\title{
Resistance to PD-1/PD-L1 blockade cancer immunotherapy: mechanisms, predictive factors, and future perspectives
}

\author{
Jin-Yu Sun ${ }^{1 \dagger}$, Dengke Zhang ${ }^{2,3 \dagger}$, Songquan $\mathrm{Wu}^{2,3}$, Min Xu ${ }^{2,3}$, Xiao Zhou ${ }^{4}$, Xiao-Jie Lu ${ }^{4^{*}}$ and Jiansong Ji ${ }^{2,3,5^{*}}$
}

\begin{abstract}
PD-1/PD-L1 blockade therapy is a promising cancer treatment strategy, which has revolutionized the treatment landscape of malignancies. Over the last decade, PD-1/PD-L1 blockade therapy has been trialed in a broad range of malignancies and achieved clinical success. Despite the potentially cure-like survival benefit, only a minority of patients are estimated to experience a positive response to PD-1/PD-L1 blockade therapy, and the primary or acquired resistance might eventually lead to cancer progression in patients with clinical responses. Accordingly, the resistance to PD-1/PD-L1 blockade remains a significant challenge hindering its further application. To overcome the limitation in therapy resistance, substantial effort has been made to improve or develop novel anti-PD-1/PD-L1 based immunotherapy strategies with better clinical response and reduced immune-mediated toxicity. In this review, we provide an overview on the resistance to PD-1/PD-L1 blockade and briefly introduce the mechanisms underlying therapy resistance. Moreover, we summarize potential predictive factors for the resistance to PD-1/PD-L1 blockade. Furthermore, we give an insight into the possible solutions to improve efficacy and clinical response. In the following research, combined efforts of basic researchers and clinicians are required to address the limitation of therapy resistance.
\end{abstract}

Keywords: PD-1/PD-L1 blockade, Cancer immunotherapy, Resistance

\section{Background}

Immunotherapy is a validated and significant cancer treatment strategy, which eliminates tumors by normalizing the anti-tumor immune responses $[1,2]$. Over the last decade, cancer immunotherapy has revolutionized the treatment landscape of malignancies and achieved clinical success, especially in immune checkpoint inhibitors [3].

\footnotetext{
*Correspondence: 189@whu.edu.cn; Ischrjj@@163.com

${ }^{\dagger}$ Jin-Yu Sun and Dengke Zhang are co-first authors.

${ }^{4}$ Department of General Surgery, The First Affiliated Hospital of Nanjing Medical University, Nanjing, China

${ }^{2}$ Key Laboratory of Imaging Diagnosis and Minimally Invasive Intervention Research, Lishui Hospital of Zhejiang University/ The Fifth Affiliated Hospital of Wenzhou Medical University/ Clinical Medicine of Center Hospital of Lishui College, Lishui 323000, China

Full list of author information is available at the end of the article
}

Programmed death-1 (PD-1) is a class of receptor expressed on the $\mathrm{T}$ cell surface, which could downregulate the immune system by abrogating $\mathrm{T}$ cell receptor-induced signals and preventing antigenmediated $\mathrm{T}$ cell activation [4]. The interaction between PD-1 and its ligand (programmed death-ligand 1, PDL1) plays an essential role in maintaining self-tolerance and avoiding autoimmune diseases. However, PD-1/PD$\mathrm{L} 1$ could also prevent the activation of $\mathrm{T}$ cells in the tumor and thus result in immune resistance [5].

PD-1/PD-L1 blockade is a breakthrough in cancer immunotherapy, and it has been trialed in a broad range of malignancies in the preclinical or clinical stage, including melanoma [6], Hodgkin's lymphoma [7], breast cancer [8,9], non-small cell lung cancer (NSCLC) [10], as well as hepatocellular carcinoma

(c) The Author(s). 2020 Open Access This article is licensed under a Creative Commons Attribution 4.0 International License, which permits use, sharing, adaptation, distribution and reproduction in any medium or format, as long as you give appropriate credit to the original author(s) and the source, provide a link to the Creative Commons licence, and indicate if changes were made. The images or other third party material in this article are included in the article's Creative Commons licence, unless indicated otherwise in a credit line to the material. If material is not included in the article's Creative Commons licence and your intended use is not permitted by statutory regulation or exceeds the permitted use, you will need to obtain permission directly from the copyright holder. To view a copy of this licence, visit http://creativecommons.org/licenses/by/4.0/ The Creative Commons Public Domain Dedication waiver (http://creativecommons.org/publicdomain/zero/1.0/) applies to the data made available in this article, unless otherwise stated in a credit line to the data. 
[11, 12]. Despite the long-term, potentially cure-like clinical benefits, therapy resistance remains a significant challenge for the further application of PD-1/ PD-L1 blockade therapy. Only a minority of patients $(20-30 \%)$ in general are estimated to experience a positive response to PD-1/PD-L1 blockade therapy [13-15], and the primary or acquired resistance might eventually lead to cancer progression in patients with clinical response [16, 17].

In this review, we provide an overview on the resistance to PD-1/PD-L1 blockade and its underlying mechanisms. Moreover, we summarize potential predictive factors for the resistance to PD-1/PD-L1 blockade. Furthermore, we give an insight into the possible solutions to improve efficacy and clinical response of PD-1/PD-L1 blockade therapy.

\section{Resistance to PD-1/PD-L1 blockade therapy}

Checkpoint inhibitors targeting PD-1 or PD-L1 could disturb the interaction between PD-1 and PD-L1, which would preserve anti-tumor properties of $\mathrm{T}$ cells, withdraw immune escape, and normalize their ability to induce tumor cell death. Currently, PD-1/PD-L1 blockade has shown sustained survival benefits in multiple malignancies and is at the forefront of cancer immunotherapy [18]. However, just as tumor cells can avoid immune evasion, several cancers may evolve to resist PD-1/PDL1 blockade therapy. Clinical evidence indicated that even for patients with tumors highly positive for PD-L1, more than $50 \%$ of them might not respond to PD-1/PDL1 blockade [19]. Due to tumor heterogeneity and many other reasons, clinical responses vary largely across different tumor entities. The objective response rate was $30-45 \%$ in melanoma [20], 15-20\% in NSCLC [21], 13\% in head and neck carcinoma [22], and 22-25\% in kidney cancer [23]. Besides, for most patients experiencing initial clinical response, acquired resistance remains another problem, which would lead to cancer progression or relapse after a few years [16, 24].

Many studies have demonstrated that anti-PD-1 therapy can significantly improve survival outcomes for patients with metastatic or unresectable melanoma [25, 26]. However, only a small number of patients (approximately $8-15 \%$ ) could achieve a complete response. In a recent phase I trial of atezolizumab (anti-PD-L1) involving 45 patients with metastatic melanoma [27], the overall response rate was $30 \%$ among 43 efficacy evaluable patients, and the median response duration was 62 months. Moreover, in another study on the long-term outcomes of melanoma patients receiving anti-PD-1 therapy, complete responses were only observed in 102 of 396 patients $(25.8 \%)$ [6]. After a median follow-up of 21.1 months, $72.1 \%$ of patients were alive without additional melanoma therapy. Additionally, in the retreated patients after disease progression, the response was only observed in $14.7 \%$ retreated patients receiving singleagent PD-1 blockade therapy, and $25.0 \%$ of patients escalated to PD-1 blockade plus ipilimumab therapy. In this cohort, most complete responses were durable with the treatment failure rate of $27 \%$ at three years, while the response to retreatment remained relatively infrequent with a response rate of $14.7 \%$ for patients with single-agent PD-1 blockade therapy [6]. Moreover, in a phase II study of pembrolizumab on 39 patients with advanced adrenocortical carcinoma, the objective response was observed in $23 \%$ of patients with a disease control rate of $52 \%$ after a median follow-up of 17.8 months [28].

Interestingly, the response rate of some malignancies is relatively high in hematological malignancies. For example, for patients with relapsed or refractory classical Hodgkin lymphoma, tislelizumab (anti-PD-1) achieved an objective response rate of $87.1 \%$ and a complete response of $62.9 \%$, in a phase II, single-arm, multicenter study [7]. Similarly, the complete response rate of camrelizumab (anti-PD-1) was $28.0 \%$, with a partial remission rate of $48.0 \%$ [29].

\section{Mechanisms underlying the resistance to PD-1/ PD-L1 blockade}

Since therapy resistance remains a significant limitation of PD-1/PD-L1 blockade in clinical practice, interest is growing in understanding the mechanisms underlying the resistance. The response to PD-1/PD-L1 blockade relies on a pre-existing immune response and determinants of adaptive immunity. Currently, multiple factors have been discovered to be involved in the efficacy of PD-1/PD-L1 blockade therapy, such as tumor immunogenicity, $\mathrm{T}$ cell function, PD-L1 expression, tumor microenvironment, and so forth.

\section{The lack of tumor antigens}

The genetic alterations are central in the oncogenic process, which could lead to tumor immunogenicity and provide an opportunity for cancer immunotherapy [17]. Tumor immunogenicity is positively associated with the ability of the $\mathrm{T}$ cell to recognize tumor cells, which is essential for the anti-tumor effect of PD-1/PD-L1 blockade. However, the lack of tumor antigen will significantly impede the recognition ability of $\mathrm{T}$ cells and eventually result in the failure of immunotherapy.

Microsatellites are prone to DNA replication errors, which will usually be repaired in normal cells [30]. However, in tumors with mismatch repair (MMR) deficiency, these errors will accumulate, which eventually result in a large number of mutations and lead to microsatellite instability (MSI) [30]. Importantly, high MSI positively contributes to increased neoantigen production, greater 
immunogenicity, and a more robust immune response [31]. Moreover, the resultant high tumor mutation burden would contribute to tumor immunogenic and enhance the response to PD-1/PD-L1 blockade therapy [32, 33].

Multiple studies have demonstrated that the tumor mutation burden is positively correlated with neoantigen burden as well as response to immunotherapy [34, 35]. For example, in colorectal cancer with MMR deficiency, which usually exhibits a high tumor mutation burden, anti-PD-1 therapy showed a higher response rate and better survival outcome compared to other subtypes with MMR proficiency [36-38]. Yarchoan et al. [38] analyzed the objective response rates of PD-1/PD-L1 blockade therapy for the corresponding tumor mutation burden in various cancers, and their results showed that the mutation burden was closely associated with the objective response rate [38].

Moreover, pancreatic cancer generally exhibits a lower mutation load compared with other solid tumors, and therefore, PD-1/PD-L1 blockade is usually ineffective for those patients and fails to improve their survival outcomes. Nevertheless, in pancreatic cancer patients harboring an MMR deficiency, they appear to be responsive to PD-1/PD-L1 blockade therapy. MMR deficiency significantly increases the somatic mutation rate, which could be translated into neoantigens and recognized by the immune system, thus making these patients responsive to PD-1/PD-L1 blockade therapy [36, 39]. Accordingly, pembrolizumab has been approved for selected cancer patients with MMR deficiency.

\section{T cell dysfunction}

Effective PD-1/PD-L1 blockade therapy relies on the T cell function, and any disruption in the processes of $\mathrm{T}$ cell immune function will result in the failure of PD-1/ PD-L1 blockade therapy. A recent review article by Ren et al. has provided an in-depth insight into the mechanisms underlying the $\mathrm{T}$ cell dysfunction-mediated resistance, with a focus on $\mathrm{T}$ cell recognition, activation, differentiation, infiltration, depletion, as well as chemotaxis [40].

Antigen presentation is a critical process for the tumor antigens identification by initial $\mathrm{T}$ cells. Beta-2microglobulin (B2M) is a significant HLA1 molecule whose mutation will hinder tumor antigen presentation and result in therapy resistance [41-43]. Zaretsky et al. [43] analyzed biopsy samples from patients with metastatic melanoma receiving pembrolizumab who exhibited disease progression after an initial tumor regression, and they found a truncating mutation in the $B 2 M$ gene. In the following research, Gettinger et al. [41] identified acquired homozygous loss or downregulation of B2M in lung patients with resistance to PD-1/PD-L1 blockade.
To further explore the role of $\mathrm{B} 2 \mathrm{M}$ in mediating resistance, they knocked out the $B 2 M$ gene in immunocompetent lung cancer mice by CRISPR technology, and the loss of $B 2 M$ resulted in the resistance to PD-1/PD-L1 blockade [41]. Additionally, B2M mutation-induced resistance primarily occurred in an environment of activated PD-1 positive $\mathrm{T}$ cell infiltration, which suggested that resistance to PD-1/PD-L1 blockade therapy might be particularly common in patients with high PD-1 positive T cell [44].

Moreover, $\mathrm{T}$ cell activation is another critical process for PD-1/PD-L1 blockade therapy. After blocking PD-1/ PD-L1, tumor cells can still counteract the activity of immune checkpoints and activate additional inhibitory pathways via expression of other immune checkpoints and their ligands within the tumor immune microenvironment [45]. For example, $\mathrm{T}$ cell immunoglobulin mucin-3 (Tim-3) is another type of immune checkpoint receptor expressed on tumor-infiltrating lymphocytes. In human head and neck squamous cell carcinoma tumorinfiltrating lymphocytes, PD-1 blockade was demonstrated to up-regulate Tim-3 expression, which inhibited $\mathrm{T}$ cells activation and contributed to Tim-3-mediated escape from PD-1 blockade in the tumor microenvironment via PI3K/Akt pathway [46].

\section{PD-1 or PD-L1}

Physiologically, interactions between PD-1 and PD-L1 block $\mathrm{T}$ cell activation pathways related to the immune response against specific antigens, and the expression of PD-1 or PD-L1 has gained importance as a significant player in regulating the response to PD-1/PD-L1 blockade therapy. PD-1 and PD-L1 are up-regulated in the tumor immune microenvironment of various malignancies, which is considered as a strategy to evade immunosurveillance and imposes a significant barrier of the anti-tumor immune response [47]. Importantly, PDL1 primarily exhibits two distinct expression patterns: on tumor cells or on tumor-infiltrating immune cells. PD-L1 expression on immune cells reflects the adaptive regulation meditated by IFN- $\gamma$, which is accompanied by increased effector $\mathrm{T}$ cells as well as tumor-infiltrating lymphocytes effector $\mathrm{T}$ cells. Differently, the expression of PD-L1 on tumor cells is less prevalent, and it indicates the epigenetically dysregulated PD-L1 gene, which is correlated with reduced immune infiltration, sclerotic or desmoplastic stroma, as well as mesenchymal molecular features [48].

Multiple studies have revealed a significantly higher objective response rate in tumor PD-L1 positive patients than PD-L1 negative subgroups, together with an improved progression-free and overall survival [21, 49-51]. Kowanetz et al. [48] observed that atezolizumab (antiPD-L1) achieved an objective response rate of $40 \%$ in 
patients with high PD-L1 levels on tumor cells alone and of $22 \%$ in those with a high expression on immune cells alone. Although these observations indicated that the functional importance of PD-L1 expression in regulating PD-1/PD-L1 blockade-induced $\mathrm{T}$ cell response, the mechanistic significance of PD-L1 on tumor cells or immune cells remains vague.

\section{Noncoding RNAs}

A large amount of microRNAs (miRNAs) and some long noncoding RNAs (lncRNAs) have emerged as players in regulating tumor immunity [52-54] and resistance to PD-1/PD-L1 blockade therapy [55].

Recently, Huber et al. [56] identified a panel of circulating miRNAs (miR-146a, miR-155, miR-125b, miR100, let-7e, miR-125a, miR-146b, miR-99b), which were associated with phenotypic and functional features of myeloid-derived suppressor cells (MDSCs) in melanoma patients. Importantly, MDSCs are a subclass of immature myeloid cells pathologically associated with cancer and play an inhibitory role against anti-tumor $\mathrm{T}$ cell immunity [57]. The transcriptional analysis showed that these miRNAs could facilitate the conversion of monocytes into MDSCs by melanoma extracellular vesicles, and the expression level of these miRNA was upregulated in circulating $\mathrm{CD}_{1} 4^{+}$monocytes and tumor samples, which was associated with myeloid cell infiltration and could predict the resistance to PD-1 blockade therapy [56].

Moreover, $\mathrm{Hu}$ et al. [58] revealed the role of oncogenic lncRNA for kinase activation (LINK-A) in losing antigenicity and evading immune checkpoints and demonstrated lncRNA-dependent antigenicity downregulation and intrinsic tumor suppression. For patients with triple-negative breast cancer and resistant to PD-1 blockade therapy, they showed up-regulated LINK-A levels and downregulated peptide-loading complex components. The analysis suggested that LINK-A expression could attenuate protein kinase A-mediated phosphorylation of the E3 ubiquitin-protein ligase TRIM71 via facilitating the crosstalk between phosphatidylinositol- [35]-trisphosphate and inhibitory G-protein-coupled receptor pathways. Consequently, LINK-A could contribute to the degradation of the antigen peptide-loading complex and up-regulate intrinsic tumor suppressors [58].

\section{Gut microbiome}

The gut microbiome is a complex system composed of more than 100 trillion microorganisms, which has been demonstrated to regulate the efficacy and toxicity of cancer immunotherapy. Many studies have reported the influence of the gut microbiome on cancer immunotherapy, and the therapeutic response of PD-1/PD-L1 blockade therapy can be improved or diminished via gut microbiome modulation.

In mice models with distinct microbiome, a significantly different response to PD-1/PD-L1 blockade therapy was observed. For example, melanoma mice with an increased Bifidobacterium species in the gut microbiome exhibited an effective response to PD-1 blockade therapy [59]. Similarly, antibiotic administration was reported to reduce the diversity and aggravate dysbiosis of the gut microbiome, thus influencing the clinical response to PD-1/PD-L1 blockade in tumor-bearing mice as well as cancer patients [60-62].. Compared to patients without antibiotic treatment, the oral antibiotic application could significantly diminish the clinical benefit of PD-1/PD-L1 blockade therapy and decrease progression-free survival and overall survival [61].

Therefore, dysbiosis of the gut microbiome is considered as one of the putative mechanisms underlying poor response to $\mathrm{PD}-1 / \mathrm{PD}-\mathrm{L} 1$ blockade therapy, and the dual-directional modulation of the gut microbiome on cancer immunotherapy is increasingly revealed. However, it is still unclear how gut microbiome regulates therapy response, and whether a specific bacterial taxa or gut microbiome as a whole plays a primary role remains largely unclear. Further research is required to provide a more in-depth understanding of the underlying mechanisms.

\section{Predictive factors for PD-1/PD-L1 blockade therapy}

Despite the clinical success achieved in PD-1/PD-L1 blockade across multiple cancers, the knowledge concerning therapy selection criteria is relatively limited. Considering the potential adverse events and high cost of immune checkpoint inhibitor agents, there is a substantial need to identify predictive factors to select patients likely to benefit from this therapy. Currently, apart from the functional status of immune cells [63-66] or tumor infiltrating lymphocytes [67], multiple factors have been identified to predict the response to PD-1/ PD-L1 blockade therapy, such as PD-1/PD-L1 expression, antigen recognition, gut microbiome, and so forth (Table 1).

\section{PD-1 or PD-L1 expression}

Inhibiting the PD-1 pathway-mediated immune suppression is the basis and premise of PD-1/PD-L1 blockade therapy. Accumulating research has suggested that PD$\mathrm{L} 1$ is a biomarker to predict therapeutic response to PD1/PD-L1 blockade across multiple tumor types [15]. For example, atezolizumab achieved overall survival benefit across all PD-L1 expression subgroups in NSCLC patients, while those with high PD-L1 expression experienced a more substantial survival benefit [76]. Currently, 
Table 1 Predictive factors for PD-1/PD-L1 blockade therapy

\begin{tabular}{|c|c|c|c|}
\hline Tumor type & Agent & Predictive factor & Reference \\
\hline Non-small cell lung cancer & Atezolizumab & PD-L1 & {$[68]$} \\
\hline Multiple cancers & Pembrolizumab & PD-L1 & [69] \\
\hline Colorectal cancer & Nivolumab & $\mathrm{MMR} / \mathrm{MSI}$ & {$[70]$} \\
\hline Urothelial carcinoma & Atezolizumab & TMB & [71] \\
\hline Urothelial carcinoma & Atezolizumab & TMB & [72] \\
\hline Urothelial cancer & Atezolizumab & TMB & [73] \\
\hline Melanoma & Anti-PD-1 therapy & Gut microbiome & [74] \\
\hline Melanoma & Anti-PD-1 therapy & Gut microbiome & [75] \\
\hline Melanoma & Anti-PD-1 therapy & Gut microbiome & {$[61]$} \\
\hline
\end{tabular}

MMR: mismatch repair; MSI: microsatellite instability; TMB: tumor mutation burden

PD-L1 testing is recommended as a predictive test for NSCLC [68], urothelial carcinoma [77], or head and neck cancers [78], and so forth.

Ott et al. [79] assessed the predictive value of PD-L1 expression in patients with advanced solid tumors receiving pembrolizumab, and the analysis showed that tumors with higher PD-L1 expression and tumor mutation burden were significantly associated with higher response rate and more prolonged progression-free survival. Heat map analysis revealed a close correlation between PD-L1 expression and a broader pattern of coregulated gene expression, which involved cytokine recruitment of T cells, $\mathrm{T}$ cell activation markers, as well as antigen presentation. Also, the regression meta-analysis demonstrated that PD-L1 expression level was positively associated with objective response rate $(P=0.018)$ as well as progression-free survival $(P=0.005,72)$.

Moreover, NCT02853305 and NCT02807636 evaluated the efficacy of pembrolizumab or atezolizumab as first-line treatment, and the current data showed reduced survival in patients with low expression of PD-L1. Accordingly, it is advised that pembrolizumab or atezolizumab should be used for adult patients with a relatively high PD-L1 expression (PD-L1 expression of $\geq 5 \%$ for atezolizumab, and a combined positive score of $\geq 10$ for pembrolizumab). However, the efficacy of PD-1/PD-L1 blockade therapy as first-line therapy for advanced urothelial carcinoma still remains unclear $[15,69]$.

Importantly, PD-L1 positive only is not a predictive factor for the response to PD-1/PD-L1 blockade, since multiple factors are involved in the PD-1/PD-L1 blockade therapy. In a study on 46 patients with metastatic melanoma receiving pembrolizumab, pre-existing $\mathrm{CD}^{+}$ $\mathrm{T}$ cells were demonstrated as a prerequisite for the tumor regression after PD-1/PD-L1 blockade therapy [80]. Besides, in advanced adrenocortical carcinoma, tumor PD-L1 expression status was not associated with therapy response [28]. Additionally, it was reported that
PD-L1 expression on tumor cells was not associated with therapy response in resected head and neck squamous cell cancer [81]. Additional investigation is required to illustrate the mechanisms accounting for the difference.

\section{Antigen recognition}

Antigen recognition plays a vital role in initiating the adaptive immune response, while the lack of tumor antigens significantly impedes the response to PD-1/PD-L1 blockade therapy.

Currently, the FDA has approved pembrolizumab to treat unresectable solid tumors with high MSI or MMR deficiency [82]. In a study on recurrent or metastatic colorectal cancer patients with MMR deficiency or high MSI, nivolumab showed an objective response rate of 31.1 , and $69 \%$ of the patients had a disease control rate of $\geq 12$ weeks, which indicated that patients with high MMR deficiency or high MSI might exhibit better responses to PD-1/PD-L1 blockade therapy [40, 83]. Interestingly, the responses of tumors with MMR-deficient are highly variable, and approximately half are resistant to PD-1/PD-L1 blockade therapy. Mandal et al. [32] revealed that MSI and the resultant mutation load were responsible for the variable response to PD-1 blockade therapy in MMR-deficiency tumors, and the response degree was significantly correlated with the degree of insertion-deletion mutation load.

Several studies have revealed the association between tumor mutation burden and the response to PD-1/PD-L1 blockade therapy [70, 71]. Mariathasan et al. [72] examined samples from patients with metastatic urothelial cancer receiving atezolizumab treatment and identified high neoantigen and tumor mutation burden as major determinants of clinical outcome. Their results showed that the tumor mutation burden was closely correlated with the response in the excluded and inflamed phenotypes. 


\section{Gut microbiome composition}

Clinical experiments on the human gut microbiome have identified several specific bacteria genres that play important roles in human immunity and can be used as prognostic biomarkers for clinical response to $\mathrm{PD}-1 / \mathrm{PD}$ L1 blockade therapy [73].

Based on the gut microbiome analysis of melanoma patients receiving PD-1 blockade, Gopalakrishnan et al. [84] found that patients with prolonged progressionfree survival showed a higher multiplicity of bacteria, and Clostridiales, Ruminococcaceae, and Faecalibacterium were abundant in therapy responders. Moreover, Matson et al. [74] evaluated the baseline stool samples from patients with metastatic melanoma before PD-1/ PD-L1 blockade treatment, and the results showed that commensal microbial composition was significantly associated with the clinical response: Bifidobacterium longum, Collinsella aerofaciens, and Enterococcus faecium were more abundant in responders. Similarly, in patients with epithelial tumors, Routy et al. [60] revealed that Akkermansiacea muciniphila and Enterococcus hirae were significantly abundant in those with better clinical response (progression-free survival $>3$ months). All these results indicate that gut microbiome composition may be a potential determinant of therapy response and might be used as a predictive factor. In the following research, more studies are needed to validate the predictive value of gut microbiome in larger cohorts and explore their efficiency in the context of various types of tumors.

\section{Future perspectives}

Immunotherapy is one of the most promising cancer treatment strategies, and it has revolutionized the landscape of cancer management over the last decade. However, together with the costly and time-consuming trial-and-error approach, the limited therapy response remains a tricky problem, which hinders the further application of PD-1/PD-L1 blockade. To overcome therapy resistance and potential adverse events, substantial effort has been made on developing novel anti-PD-1/ PD-L1 based immunotherapy strategies with better clinical response and limited immune-mediated toxicity (Figs. 1, 84).

Since the interaction between cancer and the immune system is complex and involves multiple factors, strategies in combination with multiple agents are likely to achieve better clinical outcomes compared with single-agent administration. A large number of studies have revealed that combined therapy is an effective therapeutic strategy against cancers. For example, transforming growth factor $\beta$ (TGF $\beta$ )-blocking agents concomitantly with combined PD-1/PD-L1 blockade combined provides a clinically feasible strategy to improve efficacy and reduce toxicity. Mariathasan et al. [72] revealed that metastatic urothelial cancer with up-regulated TGF $\beta$ signaling before treatment responded poorly to PD-1/PD-L1 blockade therapy. The tumors with dense collagen fibrils could trap $\mathrm{T}$ cells in the stromal compartment, thus preventing them from playing their functions. In preclinical experiments on mice with immuneexcluded phenotype, they demonstrated that the coadministration of PD-L1 blockade and TGF $\beta$-blocking agents could reduce TGF $\beta$ signaling, facilitate $\mathrm{T}$ cell infiltration, and achieve active anti-tumor immunity and tumor regression. Similarly, the combination of PD-1/PD-L1 blockade with tumor necrosis factor inhibitor [85, 86], metformin [87], anti-VEGF agents [88], or other immune checkpoint inhibitors (e.g., CXCR4 [89]) has been demonstrated as a clinically feasible strategy with improved anti-tumor efficacy and reduced toxicity.

PD-1/PD-L1 blockade usually acts on the whole host immune system instead of sitespecifically targeting tumorspecific immune cells, while nanomedicine technology provides a powerful tool to selectively deliver immune checkpoint inhibitor agents to tumors or lymphoid organs, using drug-loaded nanoparticles (usually 5 to $1,00 \mathrm{~nm}$ in diameter) [90]. Recent studies suggest that the PD-1/PD-L1 antibody could be conjugated or modified on the surface of nanoparticles, which could maintain their stability, enhance efficiency, and minimize the toxicity of PD-1/PD-L1 blockade [7, 91, 92]. For example, in gastric cancer cells, the PD-L1 blockadeconjugated nanoparticles contributed to significantly higher cellular uptake and achieved more effective inhibition of PD-L1 expression compared with the control groups [93]. Moreover, in patients with metastatic triplenegative breast cancer, the co-administration of nabpaclitaxel plus PD-L1 blockade (atezolizumab) prolonged progression-free survival [94]. Owing to the success in previous research [95], clinical trials on nanoimmunotherapy are currently underway, such as NCT03589339, and NCT03684785. These clinical trials should provide substantial evidence for the combination of nanomedicine and PD-1/PD-L1 blockade in the next few years.

Furthermore, accumulating evidence has demonstrated that gut microbiome significantly impacts the efficacy of cancer immunotherapy, which in turn indicates that the manipulation of the gut microbiome could latently affect the response to PD-1/PD-L1 blockade therapy [96-98]. Currently, antibiotic application, fecal microbiota transplantation (FMT), and diet regulation are considered as practical approaches to manipulate gut microbiome. For example, FMT from patients with a positive response to germ-free or 

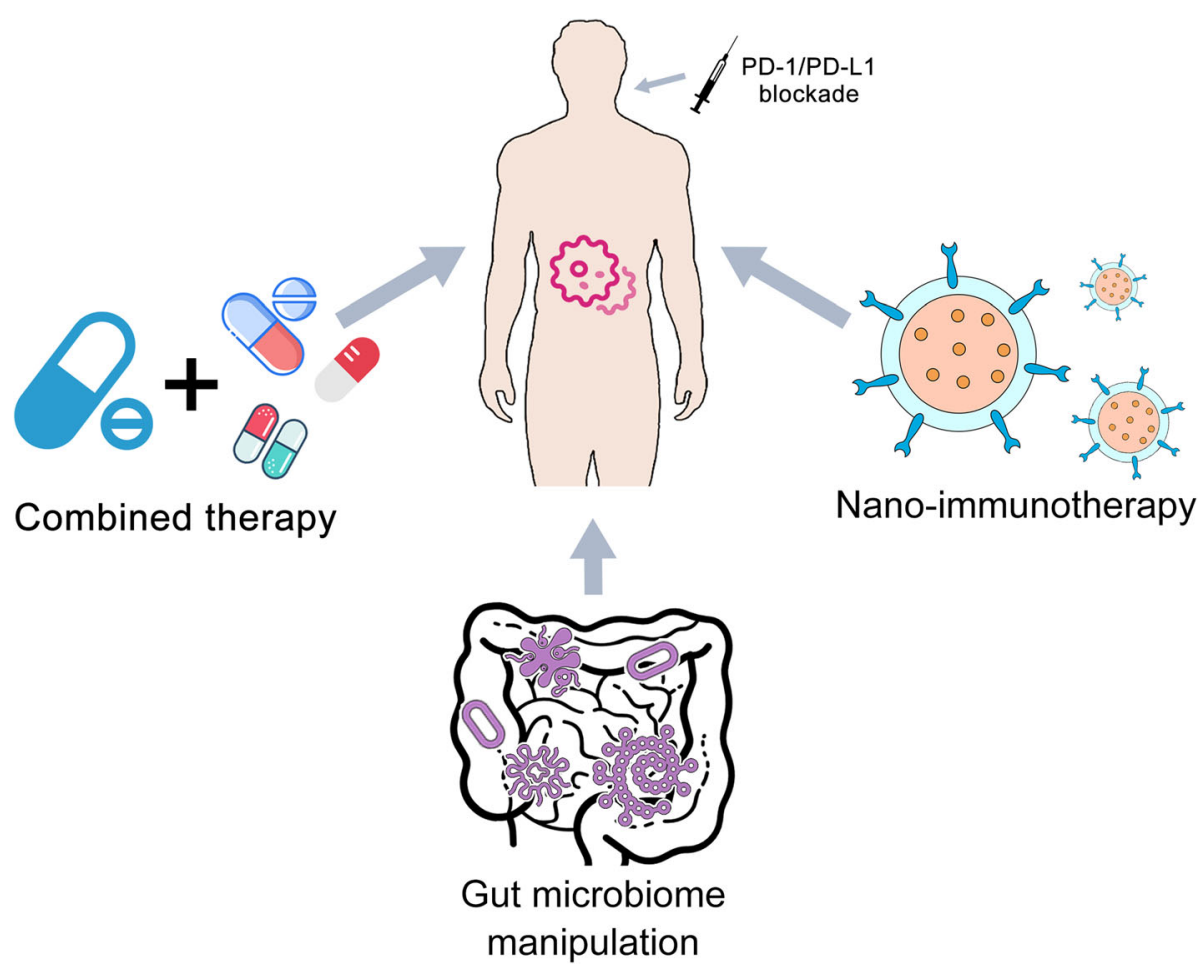

Fig. 1 Overview on the strategies to improve the resistance to PD-1/PD-L1 blockade therapy. Multiple strategies have been proposed to improve the resistance to PD-1/PD-L1 blockade therapy, including combined therapy, nano-immunotherapy, gut microbiome manipulation, and so forth

antibiotic-treated mice could improve tumor control, augment $\mathrm{T}$ cell responses, and ameliorate the antitumor effects of PD-1 blockade. In contrast, the transplantation from resistant patients did not result in improvement [60]. Similarly, responses to PD-L1 blockade are distinct in mice with different commensal microbes, and the positive response of mice with advantageous gut microbiome can be transplanted to mice with negative responses by FMT or co-housing [59].

\section{Conclusions}

Despite the success across multiple types of cancers, only a minority of patients are estimated to exhibit a positive response to $\mathrm{PD}-1 / \mathrm{PD}-\mathrm{L} 1$ blockade therapy, and the primary/acquired resistance might eventually lead to progression in patients with clinical responses. The limitation in clinical response impairs the efficacy and hinders its further application. Since the understanding of the mechanisms underlying therapy resistance remains vague, only a few therapeutic options are available for those patients. Currently, illustrating the determinants of response or resistance is significant to accelerate improving survival outcomes and developing improved treatment options for cancer patients. To better realize the therapeutic potential of PD-1/PD-L1 blockade therapy, it is essential to identify predictive biomarkers for therapy response, develop novel therapeutic strategies, and improve therapeutic strategies in combination with other agents. In the following research, combined efforts of basic researchers and clinicians are required to address the PD-1/PD-L1 blockade therapy resistance.

\section{Abbreviations}

PD-1: programmed death-1; PD-L1: programmed death-ligand 1; NSCL C: non-small cell lung cancer; MMR: mismatch repair; MSI: microsatellite instability; B2M: beta-2-microglobulin; Tim-3: T cell immunoglobulin mucin-3; miRNAs: microRNAs; IncRNAs: long noncoding RNAs; MDSCs: myeloidderived suppressor cells; TGF $\beta$ : transforming growth factor $\beta$; FMT: fecal microbiota transplantation

\section{Acknowledgments}

Not applicable.

\section{Authors' contributions}

JYS, DK Z, MX, and XZ wrote original draft preparation; SQ W, JSJ and XJ provided critical revision. All authors read and approved the final manuscript.

\section{Funding}

This study was supported by National Key Research and Development projects intergovernmental cooperation in science and technology of China (No. 2018YFE0126900 to Jiansong Ji), the Key Research and development Project of Zhejiang Province (No. 2018 C03024 to Jiansong Ji), the National Natural Science Foundation of China (81972283; 81772596 to X.L). 


\section{Availability of data and materials}

Not applicable.

\section{Ethics approval and consent to participate}

Not applicable.

\section{Consent for publication}

Not applicable.

\section{Competing interests}

The authors declare that they have no competing interests.

\section{Author details}

'The First College of Clinical Medicine, the First Affiliated Hospital of Nanjing Medical University, Nanjing Medical University, Nanjing, China. ${ }^{2}$ Key Laboratory of Imaging Diagnosis and Minimally Invasive Intervention Research, Lishui Hospital of Zhejiang University/ The Fifth Affiliated Hospital of Wenzhou Medical University/ Clinical Medicine of Center Hospital of Lishui College, Lishui 323000, China. ${ }^{3}$ College of Medicine, Lishui College, Lishui 323000, China. ${ }^{4}$ Department of General Surgery, The First Affiliated Hospital of Nanjing Medical University, Nanjing, China. ${ }^{5}$ Department of radiology, Affiliated Lishui Hospital of Zhejiang University, Lishui 323000, China.

\section{Received: 21 April 2020 Accepted: 7 August 2020}

Published online: 26 August 2020

\section{References}

1. Hellmann MD, Paz-Ares L, Bernabe Caro R, Zurawski B, Kim SW, Carcereny Costa E, et al. Nivolumab plus Ipilimumab in advanced non-small-cell lung Cancer. N Engl J Med. 2019:381(21):2020-31.

2. Niglio SA, Jia R, Ji J, Ruder S, Patel VG, Martini A, et al. Programmed Death-1 or programmed death Ligand-1 blockade in patients with platinumresistant metastatic Urothelial Cancer: a systematic review and metaanalysis. Eur Urol. 2019:76(6):782-9.

3. Sun JY, Lu XJ. Cancer immunotherapy: current applications and challenges. Cancer Lett. 2020;480:1-3.

4. Andrews $L P$, Yano $H$, Vignali DAA. Inhibitory receptors and ligands beyond PD-1, PD-L1 and CTLA-4: breakthroughs or backups. Nat Immunol. 2019; 20(11):1425-34.

5. Prestipino A, Zeiser R. Clinical implications of tumor-intrinsic mechanisms regulating PD-L1. Sci Transl Med. 2019;11(478)

6. Betof Warner A, Palmer JS, Shoushtari AN, Goldman DA, Panageas KS, Hayes $\mathrm{SA}$, et al. Long-Term Outcomes and Responses to Retreatment in Patients With Melanoma Treated With PD-1 Blockade. J Clin Oncol. 2020: JCO1901464.

7. Song Y, Gao Q, Zhang H, Fan L, Zhou J, Zou D, et al. Treatment of relapsed or refractory classical Hodgkin lymphoma with the anti-PD-1, tislelizumab: results of a phase 2, single-arm, multicenter study. Leukemia. 2020;34(2): 533-42.

8. McCart Reed AE, Kalita-De Croft P, Kutasovic JR, Saunus JM, Lakhani SR. Recent advances in breast cancer research impacting clinical diagnostic practice. J Pathol. 2019;247(5):552-62.

9. Garon EB, Hellmann MD, Rizvi NA, Carcereny E, Leighl NB, Ahn MJ, et al. Five-year overall survival for patients with advanced NonSmall-cell lung Cancer treated with Pembrolizumab: results from the phase I KEYNOTE-001 study. J Clin Oncol. 2019;37(28):2518-27.

10. Rosner S, Reuss JE, Forde PM. PD-1 blockade in early-stage lung Cancer. Annu Rev Med. 2019:70:425-35.

11. Qin S, Ren Z, Meng Z, Chen Z, Chai X, Xiong J, et al. Camrelizumab in patients with previously treated advanced hepatocellular carcinoma: a multicentre, open-label, parallel-group, randomised, phase 2 trial. Lancet Oncol. 2020.

12. Bonvalot S, Rutkowski PL, Thariat J, Carrere S, Ducassou A, Sunyach MP, et al. NBTXR3, a first-in-class radioenhancer hafnium oxide nanoparticle, plus radiotherapy versus radiotherapy alone in patients with locally advanced soft-tissue sarcoma (act.In.Sarc): a multicentre, phase 2-3, randomised, controlled trial. Lancet Oncol. 2019;20(8):1148-59.

13. Haslam A, Prasad V. Estimation of the percentage of US patients with Cancer who are eligible for and respond to checkpoint inhibitor immunotherapy drugs. JAMA Netw Open. 2019;2(5):e192535.
14. Jiang $\mathrm{Y}$, Zhao $\mathrm{X}$, Fu J, Wang $\mathrm{H}$. Progress and challenges in precise treatment of tumors with PD-1/PD-L1 blockade. Front Immunol. 2020:11:339.

15. Eckstein M, Gupta S. New insights in predictive determinants of the tumor immune microenvironment for immune checkpoint inhibition: a never ending story? Ann Transl Med. 2019;7(Suppl 3):S135.

16. Hamid O, Robert C, Daud A, Hodi FS, Hwu WJ, Kefford R, et al. Five-year survival outcomes for patients with advanced melanoma treated with pembrolizumab in KEYNOTE-001. Ann Oncol. 2019;30(4):582-8.

17. Hegde PS, Chen DS. Top 10 challenges in Cancer immunotherapy. Immunity. 2020:52(1):17-35.

18. Egen JG, Ouyang W, Wu LC. Human anti-tumor immunity: insights from immunotherapy clinical trials. Immunity. 2020;52(1):36-54.

19. Reck M, Rodriguez-Abreu D, Robinson AG, Hui R, Csoszi T, Fulop A, et al. Pembrolizumab versus chemotherapy for PD-L1-positive non-small-cell lung Cancer. N Engl J Med. 2016;375(19):1823-33.

20. Robert C, Schachter J, Long GV, Arance A, Grob JJ, Mortier L, et al. Pembrolizumab versus Ipilimumab in advanced melanoma. N Engl J Med. 2015;372(26):2521-32.

21. Borghaei H, Paz-Ares L, Horn L, Spigel DR, Steins M, Ready NE, et al. Nivolumab versus Docetaxel in advanced nonsquamous non-small-cell lung Cancer. N Engl J Med. 2015:373(17):1627-39.

22. Ferris RL, Blumenschein G Jr, Fayette J, Guigay J, Colevas AD, Licitra L, et al. Nivolumab for recurrent squamous-cell carcinoma of the head and neck. N Engl J Med. 2016;375(19):1856-67.

23. Motzer RJ, Escudier B, McDermott DF, George S, Hammers HJ, Srinivas S, et al. Nivolumab versus Everolimus in advanced renal-cell carcinoma. N Engl J Med. 2015;373(19):1803-13.

24. Bai J, Gao Z, Li X, Dong L, Han W, Nie J. Regulation of PD-1/PD-L1 pathway and resistance to PD-1/PD-L1 blockade. Oncotarget. 2017;8(66):110693-707.

25. Larkin J, Chiarion-Sileni V, Gonzalez R, Grob JJ, Cowey CL, Lao CD, et al. Combined Nivolumab and Ipilimumab or Monotherapy in untreated melanoma. N Engl J Med. 2015;373(1):23-34.

26. Robert C, Ribas A, Hamid O, Daud A, Wolchok JD, Joshua AM, et al. Durable complete response after discontinuation of Pembrolizumab in patients with metastatic melanoma. J Clin Oncol. 2018;36(17):1668-74.

27. Hamid O, Molinero L, Bolen CR, Sosman JA, Munoz-Couselo E, Kluger HM, et al. Safety, clinical activity, and biological correlates of response in patients with metastatic melanoma: results from a phase I trial of Atezolizumab. Clin Cancer Res. 2019;25(20):6061-72.

28. Raj N, Zheng Y, Kelly V, Katz SS, Chou J, Do RKG, et al. PD-1 blockade in advanced adrenocortical carcinoma. J Clin Oncol. 2020;38(1):71-80.

29. Song $Y, W u$ J, Chen $X$, Lin T, Cao J, Liu Y, et al. A single-arm, multicenter, phase II study of Camrelizumab in relapsed or refractory classical Hodgkin lymphoma. Clin Cancer Res. 2019;25(24):7363-9.

30. Baretti M, Le DT. DNA mismatch repair in cancer. Pharmacol Ther. 2018:189: 45-62.

31. Dudley JC, Lin MT, Le DT, Eshleman JR. Microsatellite instability as a biomarker for PD-1 blockade. Clin Cancer Res. 2016;22(4):813-20.

32. Mandal R, Samstein RM, Lee KW, Havel JJ, Wang H, Krishna C, et al. Genetic diversity of tumors with mismatch repair deficiency influences anti-PD-1 immunotherapy response. Science. 2019;364(6439):485-91.

33. Rizvi NA, Hellmann MD, Snyder A, Kvistborg P, Makarov V, Havel JJ, et al. Cancer immunology. Mutational landscape determines sensitivity to PD-1 blockade in non-small cell lung cancer. Science. 2015;348(6230):124-8.

34. Rooney MS, Shukla SA, Wu CJ, Getz G, Hacohen N. Molecular and genetic properties of tumors associated with local immune cytolytic activity. Cell. 2015:160(1-2):48-61.

35. Brown SD, Warren RL, Gibb EA, Martin SD, Spinelli JJ, Nelson BH, et al. Neoantigens predicted by tumor genome meta-analysis correlate with increased patient survival. Genome Res. 2014;24(5):743-50.

36. Le DT, Uram JN, Wang $H$, Bartlett BR, Kemberling $H$, Eyring AD, et al. PD-1 blockade in tumors with mismatch-repair deficiency. N Engl J Med. 2015; 372(26):2509-20.

37. Le DT, Durham JN, Smith KN, Wang H, Bartlett BR, Aulakh LK, et al. Mismatch repair deficiency predicts response of solid tumors to PD-1 blockade. Science. 2017:357(6349):409-13.

38. Yarchoan M, Hopkins A, Jaffee EM. Tumor mutational burden and response rate to PD-1 inhibition. N Engl J Med. 2017:377(25):2500-1.

39. Hilmi M, Bartholin L, Neuzillet C. Immune therapies in pancreatic ductal adenocarcinoma: where are we now? World J Gastroenterol. 2018;24(20): 2137-51. 
40. Ren D, Hua Y, Yu B, Ye X, He Z, Li C, et al. Predictive biomarkers and mechanisms underlying resistance to PD1/PD-L1 blockade cancer immunotherapy. Mol Cancer. 2020;19(1):19.

41. Gettinger S, Choi J, Hastings K, Truini A, Datar I, Sowell R, et al. Impaired HLA class I antigen processing and presentation as a mechanism of acquired resistance to immune checkpoint inhibitors in lung Cancer. Cancer Discov. 2017;7(12):1420-35

42. Sade-Feldman M, Jiao YJ, Chen JH, Rooney MS, Barzily-Rokni M, Eliane JP, et al. Resistance to checkpoint blockade therapy through inactivation of antigen presentation. Nat Commun. 2017:8(1):1136.

43. Zaretsky JM, Garcia-Diaz A, Shin DS, Escuin-Ordinas H, Hugo W, HuLieskovan S, et al. Mutations associated with acquired resistance to PD-1 blockade in melanoma. N Engl J Med. 2016;375(9):819-29.

44. Janikovits J, Muller M, Krzykalla J, Korner S, Echterdiek F, Lahrmann B, et al. High numbers of PDCD1 (PD-1)-positive T cells and B2M mutations in microsatelliteunstable colorectal cancer. Oncoimmunology. 2018;7(2):e1390640.

45. Toor SM, Sasidharan Nair V, Decock J. Elkord E. Semin Cancer Biol: Immune checkpoints in the tumor microenvironment; 2019.

46. Shayan G, Srivastava R, Li J, Schmitt N, Kane LP, Ferris RL. Adaptive resistance to anti-PD1 therapy by Tim-3 upregulation is mediated by the PI3K-Akt pathway in head and neck cancer. Oncoimmunology. 2017;6(1): e1261779.

47. Zou W, Wolchok JD, Chen L. PD-L1 (B7-H1) and PD-1 pathway blockade for cancer therapy: Mechanisms, response biomarkers, and combinations. Sci Transl Med. 2016;8(328):328rv4.

48. Kowanetz M, Zou W, Gettinger SN, Koeppen H, Kockx M, Schmid P, et al. Differential regulation of PD-L1 expression by immune and tumor cells in NSCLC and the response to treatment with atezolizumab (anti-PD-L1). Proc Natl Acad Sci U S A. 2018;115(43):E10119-E26

49. Brahmer J, Reckamp KL, Baas P, Crino L, Eberhardt WE, Poddubskaya E, et al. Nivolumab versus Docetaxel in advanced squamous-cell non-small-cell lung Cancer. N Engl J Med. 2015;373(2):123-35.

50. Weber JS, D'Angelo SP, Minor D, Hodi FS, Gutzmer R, Neyns B, et al. Nivolumab versus chemotherapy in patients with advanced melanoma who progressed after anti-CTLA-4 treatment (CheckMate 037): a randomised, controlled, open-label, phase 3 trial. Lancet Oncol. 2015; 16(4):375-84.

51. Powles T, Eder JP, Fine GD, Braiteh FS, Loriot Y, Cruz C, et al. MPDL3280A (anti-PD-L1) treatment leads to clinical activity in metastatic bladder cancer. Nature. 2014:515(7528):558-62.

52. Tang D, Zhao D, Wu Y, Yao R, Zhou L, Lu L, et al. The miR-3127-5p/p-STAT3 axis up-regulates PD-L1 inducing chemoresistance in non-small-cell lung cancer. J Cell Mol Med. 2018.

53. Zhou WY, Zhang MM, Liu C, Kang Y, Wang JO, Yang XH. Long noncoding RNA LINC00473 drives the progression of pancreatic cancer via upregulating programmed death-ligand 1 by sponging microRNA-195-5p. J Cell Physiol. 2019:234(12):23176-89.

54. Xiong G, Yang L, Chen Y, Fan Z. Linc-POU3F3 promotes cell proliferation in gastric cancer via increasing T-reg distribution. Am J Transl Res. 2015;7(11): 2262-9

55. Vishnubalaji R, Shaath H, Elango R. Alajez NM. Semin Cancer Biol: Noncoding RNAs as potential mediators of resistance to cancer immunotherapy; 2019.

56. Huber V, Vallacchi V, Fleming V, Hu X, Cova A, Dugo M, et al. Tumor-derived microRNAs induce myeloid suppressor cells and predict immunotherapy resistance in melanoma. J Clin Invest. 2018;128(12):5505-16.

57. Gabrilovich DI. Myeloid-derived suppressor cells. Cancer Immunol Res. 2017; 5(1):3-8.

58. Hu Q, Ye Y, Chan LC, Li Y, Liang K, Lin A, et al. Oncogenic IncRNA downregulates cancer cell antigen presentation and intrinsic tumor suppression. Nat Immunol. 2019;20(7):835-51.

59. Sivan A, Corrales L, Hubert N, Williams JB, Aquino-Michaels K, Earley ZM, et al. Commensal Bifidobacterium promotes antitumor immunity and facilitates anti-PD-L1 efficacy. Science. 2015:350(6264):1084-9.

60. Routy B, Le Chatelier E, Derosa L, Duong CPM, Alou MT, Daillere R, et al. Gut microbiome influences efficacy of PD-1-based immunotherapy against epithelial tumors. Science. 2018;359(6371):91-7.

61. Derosa L, Hellmann MD, Spaziano M, Halpenny D, Fidelle M, Rizvi $H$, et al. Negative association of antibiotics on clinical activity of immune checkpoint inhibitors in patients with advanced renal cell and non-small-cell lung cancer. Ann Oncol. 2018;29(6):1437-44.
62. Sun JY, Yin TL, Zhou J, Xu J, Lu XJ. Gut microbiome and cancer immunotherapy. J Cell Physiol. 2020;235(5):4082-8.

63. Tauriello DVF, Palomo-Ponce S, Stork D, Berenguer-Llergo A, Badia-Ramentol J, Iglesias $\mathrm{M}$, et al. TGFbeta drives immune evasion in genetically reconstituted colon cancer metastasis. Nature. 2018;554(7693):538-43.

64. Xue W, Li W, Zhang T, Li Z, Wang Y, Qiu Y, et al. Anti-PD1 up-regulates PDL1 expression and inhibits T-cell lymphoma progression: possible involvement of an IFN-gamma-associated JAK-STAT pathway. Onco Targets Ther. 2019;12:2079-88.

65. Jiang X, Wu H, Zhao W, Ding X, You Q, Zhu F, et al. Lycopene improves the efficiency of anti-PD-1 therapy via activating IFN signaling of lung cancer cells. Cancer Cell Int. 2019;19:68.

66. Kim KH, Cho J, Ku BM, Koh J, Sun JM, Lee SH, et al. The first-week proliferative response of peripheral blood PD-1(+)CD8(+) T cells predicts the response to anti-PD-1 therapy in solid tumors. Clin Cancer Res. 2019;25(7): 2144-54.

67. Yu PC, Long D, Liao CC, Zhang S. Association between density of tumorinfiltrating lymphocytes and prognoses of patients with gastric cancer. Medicine (Baltimore). 2018:97(27):e11387.

68. Pabani A, Butts CA. Current landscape of immunotherapy for the treatment of metastatic non-small-cell lung cancer. Curr Oncol. 2018; 25(Suppl 1):S94-S102.

69. Eckstein M, Erben P, Kriegmair MC, Worst TS, Weiss CA, Wirtz RM, et al. Performance of the Food and Drug Administration/EMA-approved programmed cell death ligand-1 assays in urothelial carcinoma with emphasis on therapy stratification for first-line use of atezolizumab and pembrolizumab. Eur J Cancer. 2019;106:234-43.

70. Rosenberg JE, Hoffman-Censits J, Powles T, van der Heijden MS, Balar AV, Necchi A, et al. Atezolizumab in patients with locally advanced and metastatic urothelial carcinoma who have progressed following treatment with platinum-based chemotherapy: a single-arm, multicentre, phase 2 trial. Lancet. 2016;387(10031):1909-20.

71. Balar AV, Galsky MD, Rosenberg JE, Powles T, Petrylak DP, Bellmunt J, et al. Atezolizumab as first-line treatment in cisplatin-ineligible patients with locally advanced and metastatic urothelial carcinoma: a single-arm, multicentre, phase 2 trial. Lancet. 2017:389(10064):67-76.

72. Mariathasan S, Turley SJ, Nickles D, Castiglioni A, Yuen K, Wang Y, et al. TGFbeta attenuates tumour response to PD-L1 blockade by contributing to exclusion of T cells. Nature. 2018;554(7693):544-8.

73. Jin $Y$, Dong $H$, Xia L, Yang Y, Zhu Y, Shen Y, et al. The diversity of gut microbiome is associated with favorable responses to anti-programmed death 1 immunotherapy in Chinese patients with NSCLC. J Thorac Oncol. 2019;14(8):1378-89.

74. Matson V, Fessler J, Bao R, Chongsuwat T, Zha Y, Alegre ML, et al. The commensal microbiome is associated with anti-PD-1 efficacy in metastatic melanoma patients. Science. 2018;359(6371):104-8

75. Riley RS, June $\mathrm{CH}$, Langer R, Mitchell MJ. Delivery technologies for cancer immunotherapy. Nat Rev Drug Discov. 2019;18(3):175-96.

76. Rittmeyer A, Barlesi F, Waterkamp D, Park K, Ciardiello F, von Pawel J, et al, Atezolizumab versus docetaxel in patients with previously treated nonsmall-cell lung cancer (OAK): a phase 3, open-label, multicentre randomised controlled trial. Lancet. 2017;389(10066):255-65.

77. Gourd E. EMA restricts use of anti-PD-1 drugs for bladder cancer. Lancet Oncol. 2018;19(7):e341

78. Yang B, Liu T, Qu Y, Liu H, Zheng SG, Cheng B, et al. Progresses and perspectives of anti-PD-1/PD-L1 antibody therapy in head and neck cancers. Front Oncol. 2018:8:563.

79. Ott PA, Bang YJ, Piha-Paul SA, Razak ARA, Bennouna J, Soria JC, et al. T-cellinflamed gene-expression profile, programmed death ligand 1 expression, and tumor mutational burden predict efficacy in patients treated with Pembrolizumab across 20 cancers: KEYNOTE-028. J Clin Oncol. 2019;37(4): 318-27.

80. Tumeh PC, Harview CL, Yearley JH, Shintaku IP, Taylor EJ, Robert L, et al. PD1 blockade induces responses by inhibiting adaptive immune resistance. Nature. 2014;515(7528):568-71.

81. Kim HR, Ha SJ, Hong MH, Heo SJ, Koh YW, Choi EC, et al. PD-L1 expression on immune cells, but not on tumor cells, is a favorable prognostic factor for head and neck cancer patients. Sci Rep. 2016;6:36956.

82. Marcus L, Lemery SJ, Keegan P, Pazdur R. FDA approval summary: Pembrolizumab for the treatment of microsatellite instability-high solid tumors. Clin Cancer Res. 2019;25(13):3753-8. 
83. Overman MJ, McDermott R, Leach JL, Lonardi S, Lenz HJ, Morse MA, et al. Nivolumab in patients with metastatic DNA mismatch repair-deficient or microsatellite instability-high colorectal cancer (CheckMate 142): an openlabel, multicentre, phase 2 study. Lancet Oncol. 2017;18(9):1182-91.

84. Gopalakrishnan V, Spencer CN, Nezi L, Reuben A, Andrews MC, Karpinets TV, et al. Gut microbiome modulates response to anti-PD-1 immunotherapy in melanoma patients. Science. 2018;359(6371):97-103.

85. Perez-Ruiz E, Minute L, Otano I, Alvarez M, Ochoa MC, Belsue V, et al. Prophylactic TNF blockade uncouples efficacy and toxicity in dual CTLA-4 and PD-1 immunotherapy. Nature. 2019;569(7756):428-32.

86. Bertrand F, Montfort A, Marcheteau E, Imbert C, Gilhodes J, Filleron T, et al. TNFalpha blockade overcomes resistance to anti-PD-1 in experimental melanoma. Nat Commun. 2017;8(1):2256.

87. Scharping NE, Menk AV, Whetstone RD, Zeng X, Delgoffe GM. Efficacy of PD-1 blockade is potentiated by metformin-induced reduction of tumor hypoxia. Cancer Immunol Res. 2017;5(1):9-16.

88. Munn LL, Jain RK. Vascular regulation of antitumor immunity. Science. 2019; 365(6453):544-5

89. Du FY, Zhou QF, Sun WJ, Chen GL. Targeting cancer stem cells in drug discovery: current state and future perspectives. World I Stem Cells. 2019; 11(7):398-420.

90. Shi J, Kantoff PW, Wooster R, Farokhzad OC. Cancer nanomedicine: progress, challenges and opportunities. Nat Rev Cancer. 2017;17(1):20-37.

91. Irvine DJ. Dane EL. Nat Rev Immunol: Enhancing cancer immunotherapy with nanomedicine; 2020.

92. Chen Q, Xu L, Liang C, Wang C, Peng R, Liu Z. Photothermal therapy with immune-adjuvant nanoparticles together with checkpoint blockade for effective cancer immunotherapy. Nat Commun. 2016;7:13193.

93. Xu S, Cui F, Huang D, Zhang D, Zhu A, Sun X, et al. PD-L1 monoclonal antibody-conjugated nanoparticles enhance drug delivery level and chemotherapy efficacy in gastric cancer cells. Int J Nanomedicine. 2019;14: $17-32$.

94. Schmid P, Adams S, Rugo HS, Schneeweiss A, Barrios $\mathrm{CH}$, Iwata $\mathrm{H}$, et al. Atezolizumab and nab-paclitaxel in advanced triple-negative breast Cancer. N Engl J Med. 2018;379(22):2108-21.

95. Liu X, Feng Z, Wang C, Su Q, Song H, Zhang C, et al. Co-localized delivery of nanomedicine and nanovaccine augments the postoperative cancer immunotherapy by amplifying T-cell responses. Biomaterials. 2020;230: 119649.

96. Inamura K. Roles of microbiota in response to cancer immunotherapy. Semin Cancer Biol. 2020

97. Gopalakrishnan V, Helmink BA, Spencer CN, Reuben A, Wargo JA. The influence of the gut microbiome on Cancer, immunity, and Cancer immunotherapy. Cancer Cell. 2018;33(4):570-80.

98. Panebianco C, Andriulli A, Pazienza V. Pharmacomicrobiomics: exploiting the drug-microbiota interactions in anticancer therapies. Microbiome. 2018; 6(1):92.

\section{Publisher's Note}

Springer Nature remains neutral with regard to jurisdictional claims in published maps and institutional affiliations.

Ready to submit your research? Choose BMC and benefit from:

- fast, convenient online submission

- thorough peer review by experienced researchers in your field

- rapid publication on acceptance

- support for research data, including large and complex data types

- gold Open Access which fosters wider collaboration and increased citations

- maximum visibility for your research: over $100 \mathrm{M}$ website views per year

At BMC, research is always in progress.

Learn more biomedcentral.com/submissions 